\title{
Lattice Tilt Mapping using Full Field Diffraction X-Ray Microscopy at ID01 ESRF
}

$\underline{\text { Tao Zhou }}^{1}$, Tomas Stankevic ${ }^{2}$, Andrea Troian ${ }^{3}$, Zhe Ren $^{3}$, Zhaoxia Bi $^{3}$, Jonas Ohlsson ${ }^{3}$, Lars Samuelson $^{3}$, Jan Hilhorst ${ }^{1}$, Tobias Schulli ${ }^{1}$, Anders Mikkelsen ${ }^{3}$, Olivier Balmes ${ }^{2, *}$

1. European Synchrotron Radiation Facility, 71 Avenue des Martyrs, 38000 Grenoble, France

2. MAX IV Laboratory, Fotongatan 2, 22592 Lund, Sweden

3. Lund University, Box 117, SE-221 00 Lund, Sweden

* Corresponding author, olivier.balmes@maxiv.lu.se

In this paper we demonstrate lattice tilt mapping on arrays of patterned InGaN pyramids using Full Field Diffraction X-ray Microscopy (FFDXM) on the ID01 beamline at the European Synchrotron (ESRF)[1]. The data was acquired during one of the first user experiments since the instrument was open to public in April 2017. The schematic of the FFDXM is shown in Fig. 1. The beamline typically operates around

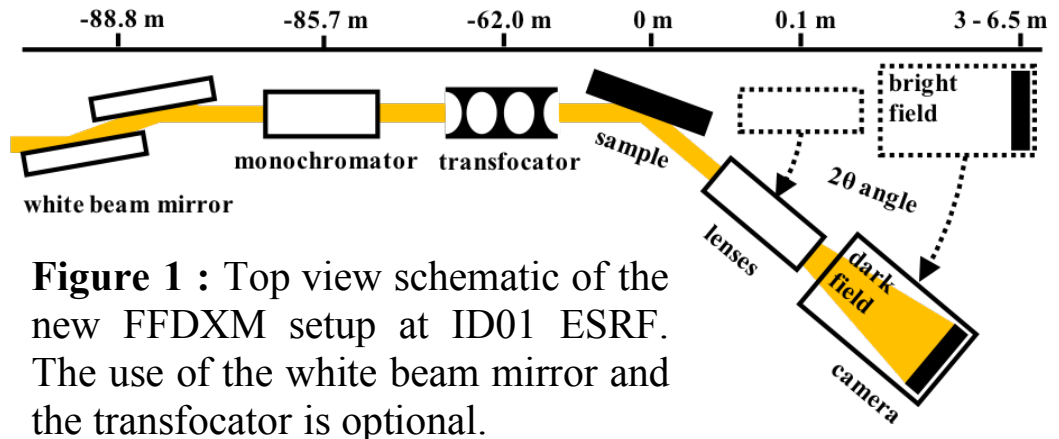

8 or $20 \mathrm{keV}$. A white beam mirror is used to reject the higher harmonics, and to pre-focus the beam in the horizontal direction. A Be transfocator is used to further condense the beam in both directions while preserving a small convergence angle $(<0.02 \mathrm{mrad})$ for maximizing the angular resolution in diffraction experiments. The flux density at the sample is $1 \times 10^{11} \mathrm{ph} / \mathrm{sec}$ for an area of $100 \times 100 \mu \mathrm{m}^{2}$ with the Si double crystal monochromator, and can be increased to $2 \times 10^{12}$ $\mathrm{ph} / \mathrm{sec}$ with the $\mathrm{Ni}_{94} \mathrm{~V}_{6}$ multilayer monochromator at the expense of energy (angular) resolution. The choice of the objective lens depends largely on the sample. The Be Compound Refractive Lens (CRLs) offers a large Field of View (FoV) of $230 \times 230 \mu \mathrm{m}^{2}$ at $8 \mathrm{keV}$. The SU-8 CRLs allows the imaging of structures underneath thick substrates or through liquids at $20 \mathrm{keV}$, with a smaller FoV of $100 \times 100 \mu \mathrm{m}^{2}$ [2]. An Andor sCMOS camera $(6.5 \mu \mathrm{m}$ pixel size $)$, mounted behind $15 \mu \mathrm{m}$ of Gadox scintillator, is installed in a vacuum pipe with adjustable distance to the sample. At $6.5 \mathrm{~m}$ and with a typical CRL focal length of $10 \mathrm{~cm}$, one obtains a magnification ratio of $65 \times$ and an effective pixel size of $100 \mathrm{~nm}$, which is comparable to the $\sim 150 \mathrm{~nm}$ spatial resolution delivered by the CRLs. For better resolutions, the $\mathrm{WSi}_{2} / \mathrm{Si}$ Multilayer Laue Lens (MLL) is used instead [3]. The MLL offers a sub-70nm resolution and a $\sim 2$-fold increase in efficiency compared to CRLs, at the cost of an even smaller FoV and shorter working distance. Both the lenses and the vacuum pipe hosting the camera are capable of rotating around the sample, to perform magnified dark field imaging using diffracted beam of up to $90^{\circ}$ two-theta angle.

InGaN pyramids were grown with selective area growth by metal-organic vapor phase epitaxy on GaN on sapphire substrates with $\mathrm{SiN}$ as a growth mask [4]. Such InGaN pyramids can be used as a high quality template for optoelectronic devices (such as LEDs) having indium-rich InGaN active layers with improved lattice match and reduced quantum-confined Stark effect. However, photo-luminescence (PL) measurements indicated that the optical performance depends heavily on the opening and pitch of the growth mask. To understand this dependence and its structural origin, a special sample has been made with several areas patterned with different opening and pitch values.

The result of rocking scans with FFDXM imaging is shown in Fig. 2A-D. At different $\theta$ angles, different pyramids were observed, indicating a strong variation (of as much as $1^{\circ}$ ) in the lattice tilt angle among 
the pyramids. The integrated intensity of the rocking curve itself is, in fact, a tilt histogram of the pyramids in the plane of rocking. With FFDXM, it is possible to obtain both statistical and localized tilt information, simultaneously on 4 adjacent areas patterned with different parameters. The composite tilt map in Fig. 2E. shows that the bottom right region with the largest pitch and opening has the largest tilt variation and the lowest yield (dark areas). With the same pitch and smaller opening (bottom left), both the yield and the homogeneity were significantly improved. This is understood as due to defect confinement in structures of smaller sizes. The majority of the pyramids in this case are either orange, yellow and green, with some occurrences of red pyramids (different fake colors here indicate different tilt angles). Interestingly, a better homogeneity is achieved by keeping the same opening but reducing the pitch (top right). The majority of the pyramids in this case are either yellow or green, with no occurrences at all of the red pyramids. The observed improvement in structural homogeneity is in good agreement with the improvement of optical performance in PL measurements. Finally, pyramids of the highest quality were observed on the pattern with at the same time smaller pitch and opening (top left).

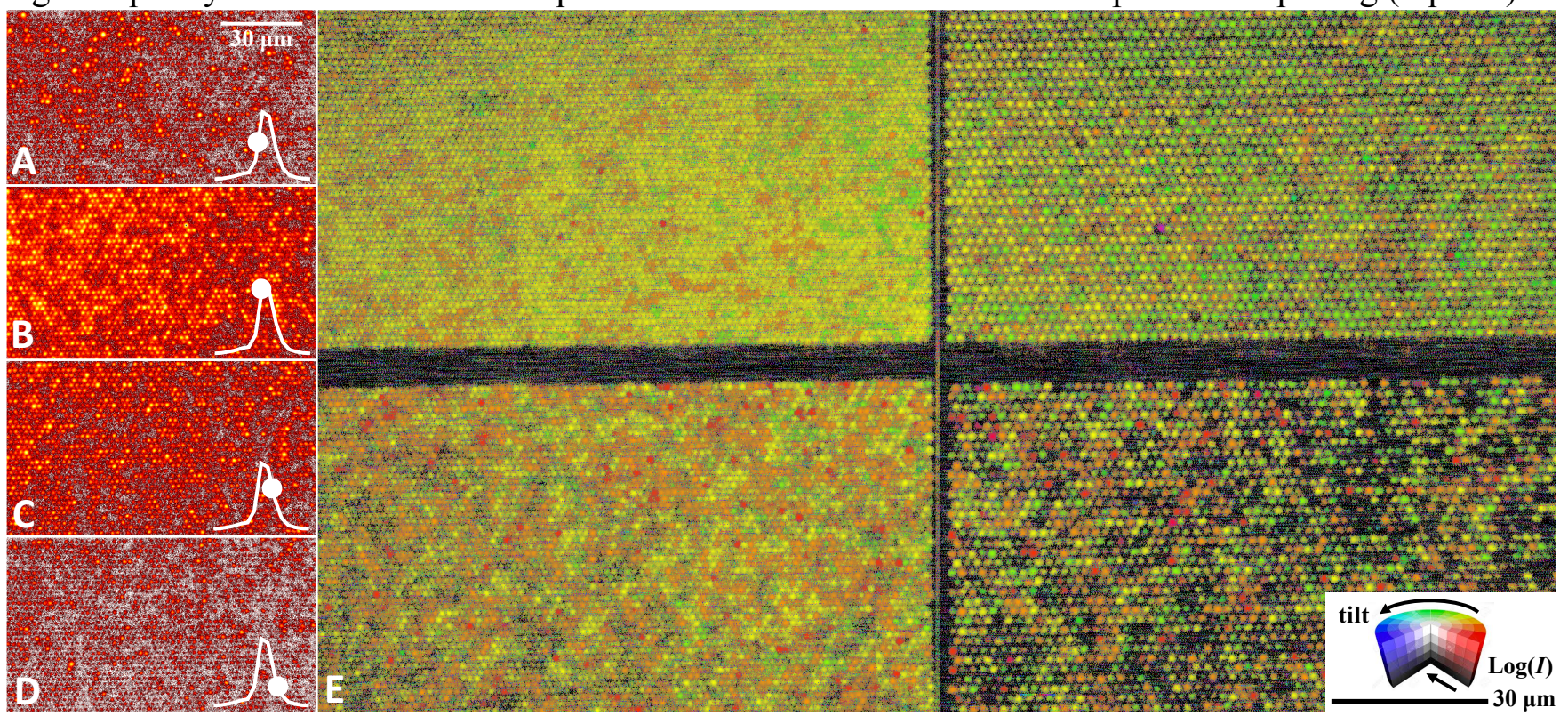

Figure 2 : (A-D) Cropped FFDXM image on a small area of the sample acquired at different angles on the rocking curve (inset). The fake color scale represents the intensity in log scale. (E) Composite lattice tilt map of the nano-pyramids at the junction of 4 areas patterned with different parameters. The actual image (FoV) is 4 times larger, but is cropped to fit in the publication. Here, different tilt angles are shown as different hues while the log of the intensity is used for saturation in the HSV colormap (inset).

FFDXM complements TXM as it offers structural information of the sample. The same measurement and data analysis strategy have also been applied to radial scans to compose lattice strain maps with a resolution of $\Delta a / a \sim 10^{-5}$. Moreover, as is true of other X-ray microscopy techniques, FFDXM is capable to probing structures buried hundreds of microns beneath the surface, which is inaccessible to electron microscopy methods. Lastly, the fast acquisition and large FoV makes the technique suitable for in situ and operando experiments. The details of these applications are omitted here to conserve space.

Reference:

[1] J. Hilhorst et al. J. Appl. Crystal. 47, 1882 (2014).

[2] F. Marschall, et al., Opt. Express 24, 10880 (2016).

[3] S. Niese, et al., Opt. Express 22, 20008 (2014).

[4] Z. Bi, et al. J. Appl. Phys. 123, 25102 (2018). 